\section{Padrões de utilização de atendimento médico- ambulatorial no Brasil entre usuários do Sistema Único de Saúde, da saúde suplementar e de serviços privados}

\author{
Patterns in the use of outpatient care in Brazil \\ by patients treated through the Brazilian Unified \\ National Health System, private health insurance, \\ and out-of-pocket medical care
}

\author{
Los patrones de uso de atención médica \\ ambulatoria en Brasil entre el Sistema Único \\ de Salud, la salud y los servicios privados \\ suplementarios
}

\author{
Alitéia Santiago Dilélio 1 \\ Elaine Tomasi 1 \\ Elaine Thumé 2 \\ Denise Silva da Silveira 3 \\ Fernando Carlos Vinholes Siqueira 4 \\ Roberto Xavier Piccini 3 \\ Suele Manjourany Silva 1 \\ Bruno Pereira Nunes 1 \\ Luiz Augusto Facchini 1
}

\author{
1 Programa de Pós-graduação \\ em Epidemiologia, \\ Universidade Federal de \\ Pelotas, Pelotas, Brasil. \\ 2 Programa de Pós- \\ graduação em Enfermagem, \\ Universidade Federal de \\ Pelotas, Pelotas, Brasil. \\ 3 Faculdade de Medicina, \\ Universidade Federal de \\ Pelotas, Pelotas, Brasil. \\ 4 Programa de Pós-graduação \\ em Educação Física, \\ Universidade Federal de \\ Pelotas, Pelotas, Brasil. \\ Correspondência \\ A. S. Dilélio \\ Programa de Pós-graduação \\ em Epidemiologia, \\ Universidade Federal de \\ Pelotas. \\ Av. Duque de Caxias 250, \\ Pelotas, RS 96030-000, Brasil. \\ aliteia@gmail.com
}

\begin{abstract}
Indicators of supply, access, and use of healthcare are helpful for characterizing services, planning activities, and organizing demand. This study describes patterns of use of outpatient care and the associations with demographic and socioeconomic factors, morbidity, population size of the municipality and region, and administrative and financial classification of the provider. This was a population-based cross-sectional study with a sample of 12,402 Brazilian adults from 20 to 59 years of age living in urban areas of 100 municipalities from five regions. Prevalence of outpatient care in the three months prior to the interview was $34.6 \%$. The Brazilian Unified National Health System (SUS) accounted for more than half (53.6\%) of the cases, health insurance was used by $34 \%$ of the sample, and out-of-pocket services $12.4 \%$, regardless of region, population size, and morbidity profile. Patterns of use of health services remain socially determined, resulting from the supply, demographic and socioeconomic characteristics, and health profile of users.
\end{abstract}

Ambulatory Care; Unified Health System; Health Evaluation

\section{Resumo}

Indicadores de oferta, acesso e utilização são úteis para caracterizar os serviços, planejar ações e organizar a demanda. Este estudo descreve padrões de utilização de atendimento médico-ambulatorial e associação com variáveis sociodemográficas, de morbidade, porte do município e região, de acordo com a natureza jurídica do serviço. Tratase de um estudo transversal de base populacional com 12.402 adultos brasileiros entre 20 e 59 anos, residentes nas áreas urbanas de 100 municípios nas cinco regiões brasileiras. A prevalência de atendimento médico-ambulatorial nos três meses anteriores à entrevista foi de 34,6\%. O Sistema Único de Saúde foi responsável por mais da metade $(53,6 \%)$ dos atendimentos, algum convênio de saúde foi utilizado por $34 \%$ da amostra e os serviços privados por $12,4 \%$, independentemente da região, do porte populacional e da morbidade referida. Os padrões de utilização de serviços de saúde continuam socialmente determinados, resultando da oferta, das características sociodemográficas e do perfil de saúde dos usuários.

Assistência Ambulatorial; Sistema Único de Saúde; Avaliação em Saúde 


\section{Introdução}

A avaliação dos serviços de saúde ganha destaque nos últimos anos pela importância estratégica na qualificação da assistência e planejamento do sistema de saúde 1. Indicadores de oferta, acesso e utilização são úteis para caracterizar os serviços, planejar ações, organizar a demanda e redimensionar os esforços do sistema de saúde a fim de contemplar as necessidades de saúde da população 2,3,4,5,6,7,8.

No Brasil, o Sistema Único de Saúde (SUS) propõe o acesso universal, igualitário e equitativo a todos os indivíduos residentes no território nacional. Cerca de um quinto (18\%) dos serviços de saúde disponíveis à população é ofertado pelas das unidades básicas de saúde (Cadastro Nacional de Estabelecimentos de Saúde - CNES. http://cnes.datasus.gov.br/, acessado em 12/ Jan/2013), mas a precariedade da infraestrutura, aliada à falta de recursos materiais e humanos, podem induzir parte da população a buscar alternativas para acessar o sistema de saúde e garantir a assistência, utilizando convênios e serviços privados 9,10,11,12.

A utilização de serviços de saúde é resultante da oferta, das características sociodemográficas e do perfil de saúde dos usuários ${ }^{13}$. Nas últimas décadas é possível identificar iniciativas do governo federal que objetivam construir e induzir processos que ampliem e assegurem o acesso, melhorem a qualidade da atenção, garantam o vínculo entre profissional e usuário e promovam a resolubilidade da assistência em todos os níveis de atenção. O Programa Nacional de Avaliação de Serviços de Saúde e Programa Nacional de Melhoria do Acesso e da Qualidade da Atenção Básica 1,14 são exemplos dessas iniciativas.

Há um importante debate sobre a capacidade do sistema de saúde público garantir uma atenção integral a indivíduos e coletividades 15 . A programação de serviços que considere as necessidades de saúde da população seria uma alternativa para superar as limitações decorrentes da programação com base na oferta. Deve-se levar em conta, ainda, a economia de escala dos serviços em relação à sua qualidade sem deixar de ter em conta o nível de acesso dos cidadãos 16 .

A Política Nacional de Saúde dispõe sobre as condições para a promoção, proteção e recuperação da saúde e a organização e o funcionamento dos serviços 17. A Portaria MS no 687/ 200618 tem como objetivo promover a qualidade de vida e reduzir a vulnerabilidade e os riscos à saúde relacionados aos seus determinantes $\mathrm{e}$ condicionantes.

Nesse aspecto, destaca-se a importância das estratégias de monitoramento e avaliação para acompanhar o desempenho dos serviços em relação às prioridades e intervenções e a adesão da população aos cuidados de saúde 17 .

Para contribuir com esse debate, o artigo descreve os padrões de utilização de atendimento médico-ambulatorial no Brasil, analisando as diferenças por natureza do serviço.

\section{Metodologia}

Para estudar o desfecho "utilização de atendimento médico ambulatorial”, realizou-se um estudo transversal de base populacional com uma amostra de adultos entre 20 e 59 anos, residentes em áreas urbanas de 100 municípios de 23 estados brasileiros (Acre, Alagoas, Bahia, Ceará, Espírito Santo, Goiás, Maranhão, Mato Grosso, Mato Grosso do Sul, Minas Gerais, Pará, Paraíba, Paraná, Pernambuco, Piauí, Rio de Janeiro, Rio Grande do Norte, Rio Grande do Sul, Rondônia, Santa Catarina, São Paulo, Sergipe e Tocantins) nas cinco regiões geopolíticas (Centro-oeste, Nordeste, Norte, Sudeste e Sul).

Estimou-se uma amostra de 12.298 adultos para investigar associações, com nível de significância de 5\%, poder de $80 \%$, já acrescida de $10 \%$ para perdas, $15 \%$ para fatores de confusão, um efeito de delineamento de 1,7, considerando a baixa renda como fator de exposição. Tal amostra também seria suficiente e adequada para comparar diferenças por sexo, idade e problemas de saúde referentes à utilização de serviços de saúde individuais.

A amostra representativa da população adulta urbana foi localizada por um processo amostral complexo em múltiplos níveis, a exemplo do que foi utilizado em outros inquéritos 19,20, atentando para o porte populacional, os setores censitários e os domicílios. Os municípios e os setores censitários foram selecionados aleatoriamente, tendo sido utilizada a malha oficial do censo populacional do ano de 2000 (Instituto Brasileiro de Geografia e Estatística - IBGE. Censo Demográfico 2000. http://www.ibge.gov.br) para a seleção dos setores. Os municípios foram agregados segundo o tamanho da população e posteriormente selecionados aleatoriamente. O sorteio dos setores censitários também foi realizado por meio da alocação aleatória de acordo com a proporção de setores válidos e o tamanho da população. Em cada um dos 638 setores selecionados, 10 domicílios eram visitados, seguindo um "salto" sistemático entre as residências, esperando-se encontrar 17 adultos por setor.

Todos os adultos residentes nos domicílios foram elegíveis para as entrevistas e, na ausência ou impossibilidade do indivíduo em responder ao questionário, foram utilizados informantes-chave, 
que deveriam ter idade maior ou igual a 16 anos e dispor de informações sobre a situação de saúde e a busca/utilização de serviços de saúde.

As entrevistas foram realizadas de agosto de 2008 a fevereiro de 2009 durante os turnos da manhã e tarde, por 55 entrevistadores, selecionados e capacitados por 40 horas. Em caso de recusa, o supervisor era comunicado, fazendo uma tentativa de reversão. Em caso de domicílios fechados, cujos vizinhos informassem morar elegíveis, o entrevistador fazia uma tentativa em outro momento do trabalho de campo.

Foi utilizado computador de mão (PDA - personal digital assistant, equipado com GPS - global positioning system) com questionário padronizado e pré-testado, contendo variáveis socioeconômicas, demográficas e de saúde. Uma vez coletados, os dados eram armazenados em computadores portáteis e transferidos, via Internet, à coordenação do estudo.

As análises aqui apresentadas fazem parte de um estudo maior, cujo principal objetivo foi caracterizar o acesso, a utilização e a qualidade da rede de saúde no Brasil. Financiada pelo Ministério da Saúde, a pesquisa já deu origem a outras publicações 21,22,23 e conta com outras sendo produzidas.

O desfecho foi obtido por uma pergunta direta, com resposta dicotômica (sim ou não): "Desde $<$ TRÊS MESES ATRÁS> o Sr(a) foi atendido(a) por algum médico?". Essa utilização de serviços médicos referiu-se a atendimentos ambulatoriais, incluindo unidades básicas de saúde (UBS), estabelecimento de especialidades médicas sem internação 20 e consultórios.

A principal variável independente foi a natureza do prestador do serviço, estudada em três categorias: convênio, serviço privado e SUS. As demais variáveis consideradas foram: região $(\mathrm{CO} / \mathrm{N} /$ $\mathrm{NE} / \mathrm{SE} / \mathrm{S}$ ), porte populacional do município (até $30.000 / 30.001$ a $100.000 / 100.001$ a $1.000 .000 />$ 1.000 .000 de habitantes), classificação econômica da Associação Brasileira de Empresas de Pesquisa (ABEP. http://www.abep.org) (A e B/C/D e E), renda per capita em salários mínimos (até 0,3/de 0,4 $\mathrm{a}<1 / 1,0$ e mais), escolaridade em anos completos (nenhum/1 a $4 / 5$ a $8 / 9$ a 11/12 ou mais), sexo (masculino/feminino), idade em anos completos ( 20 a 29/30 a 39/40 a 49/50 a 59), cor da pele autorreferida (branca/preta/outras - composta pelas categorias mestiça, amarela e indígena), situação conjugal (vive com companheiro - sim/não) e morbidade referida com base em diagnóstico médico (hipertensão arterial sistêmica/diabetes mellitus/problemas de nervos - sim/não). A renda familiar foi agrupada pela distribuição dos valores da variável numérica, de forma a garantir um mínimo de homogeneidade entre as categorias para as análises.
Para descrever as características do atendimento médico ambulatorial, considerou-se: gasto com atendimento, medicação e exame (sim/não), local do atendimento (município de residência - sim/não), prescrição de medicação (sim/não), solicitação de exame (sim/não), encaminhamento para outro serviço (sim/não), satisfação com o tempo de espera e com o atendimento (péssimo/ ruim/regular/bom/ótimo), explicação sobre o motivo do atendimento (sim/não) e o que aconteceu após o atendimento (piorou/continua como antes/melhorou um pouco/melhorou bastante/ curou-resolveu). Os motivos para buscar o atendimento foram coletados em uma variável aberta pela qual foram listadas as respostas mais frequentes: revisar ou acompanhar problema, renovar receita, fazer pré-natal, realizar exame pré-câncer e de próstata.

Para o controle de qualidade, $8 \%$ das entrevistas foram selecionadas aleatoriamente e refeitas, verificando-se a concordância entre todas as variáveis-filtro dos diferentes blocos do instrumento, por intermédio da estatística kappa. Todas apresentaram índice superior a 0,7, indicando ótima concordância.

Todas as análises foram feitas com o pacote estatístico Stata 11.0. (Stata Corp., College Station, Estados Unidos) e incluíram o cálculo das proporções e seus respectivos intervalos de $95 \%$ de confiança.

O protocolo do estudo foi aprovado pelo Comitê de Ética em Pesquisa da Faculdade de Medicina da Universidade Federal de Pelotas (no 152/07, de 23 de novembro de 2007) e o consentimento informado foi obtido de todos os entrevistados. Os autores declaram não haver nenhum tipo de conflito de interesses no presente estudo.

\section{Resultados}

Descrição da amostra e da utilização de atendimento médico ambulatorial

Participaram do estudo 12.402 adultos. A proporção de perdas e recusas foi de $9,8 \%$, totalizando 1.354 indivíduos.

As mulheres representaram $55,1 \%$ da amostra. Cerca de um terço $(31,8 \%)$ dos adultos encontrava-se na faixa etária de 20 a 29 anos, com idade média de 37,4 anos (DP $\pm 11,5$ ), e $39,7 \%$ referiram cor da pele branca (Tabela 1).

Mais da metade (51,5\%) dos adultos encontrava-se na classificação econômica C e $42 \%$ possuíam renda per capita superior a um salário mínimo. Cerca de um terço $(34,5 \%)$ dos indivíduos completou entre 9 e 11 anos de estudo. 
Tabela 1

Descrição da amostra e caracterização do atendimento médico de acordo com variáveis de contexto, socioeconômicas , demográficas e de saúde da população estudada. Brasil, 2009.

\begin{tabular}{|c|c|c|c|}
\hline \multirow[t]{2}{*}{ Variável } & \multirow{2}{*}{$\begin{array}{l}\text { Amostra total } \\
\qquad n^{*}(\%)\end{array}$} & \multicolumn{2}{|c|}{ Utilização de atendimento médico } \\
\hline & & $\mathrm{n} * *$ & $\%(I C 95 \%)$ \\
\hline \multicolumn{4}{|c|}{ 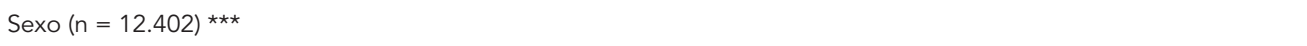 } \\
\hline Masculino & $5.574(44,9)$ & 1.385 & $25,1(24,0-26,3)$ \\
\hline Feminino & $6.828(55,1)$ & 2.856 & $42,1(40,9-43,3)$ \\
\hline \multicolumn{4}{|c|}{ Idade (anos) $(\mathrm{n}=12.371)$} \\
\hline $20-29$ & $3.938(31,8)$ & 1.117 & $28,6(27,2-30,0)$ \\
\hline $30-39$ & $3.114(25,2)$ & 1.045 & $33,8(32,2-35,5)$ \\
\hline $40-49$ & $2.958(23,9)$ & 1.078 & $36,7(35,0-38,5)$ \\
\hline $50-59$ & $2.361(19,1)$ & 993 & $42,4(40,4-44,4)$ \\
\hline \multicolumn{4}{|c|}{ Cor da pele $(n=11.979)$} \\
\hline Branca & $4.752(39,7)$ & 1.766 & $37,6(36,2-39,0)$ \\
\hline Preta & $770(6,4)$ & 267 & $34,9(31,6-38,4)$ \\
\hline Outras & $6.457(53,9)$ & 2.099 & $32,7(31,5-33,9)$ \\
\hline \multicolumn{4}{|c|}{ Escolaridade (anos) $(n=12.336)$} \\
\hline Nenhum & $774(6,3)$ & 240 & $31,4(28,1-34,8)$ \\
\hline $1-4$ & $1.939(15,7)$ & 687 & $35,6(33,5-37,8)$ \\
\hline $5-8$ & $3.530(28,6)$ & 1.160 & $33,3(31,7-34,9)$ \\
\hline $9-11$ & $4.253(34,5)$ & 1.405 & $33,2(31,8-34,6)$ \\
\hline 12 e mais & $1.840(14,9)$ & 730 & $40,2(38,0-42,5)$ \\
\hline \multicolumn{4}{|c|}{ Renda (salários mínimos) ( $n=11.767$ ) } \\
\hline Até 0,3 & $2.547(21,7)$ & 750 & $29,5(26,2-32,9)$ \\
\hline De $0,4 a<1,0$ & $4.276(36,3)$ & 1.392 & $32,6(30,2-35,1)$ \\
\hline $1,0 \mathrm{e}+$ & $4.944(42,0)$ & 1.894 & $38,5(36,3-40,7)$ \\
\hline \multicolumn{4}{|c|}{ Classe econômica ( $n=11.674)$} \\
\hline $\mathrm{A} / \mathrm{B}$ & $3.302(28,3)$ & 1.339 & $40,7(39,2-42,4)$ \\
\hline C & $6.013(51,5)$ & 1.988 & $33,2(32,0-34,4)$ \\
\hline $\mathrm{D} / \mathrm{E}$ & $2.359(20,2)$ & 674 & $28,6(26,8-30,5)$ \\
\hline \multicolumn{4}{|c|}{ Vive com companheiro $(n=12.393)$} \\
\hline Não & $4.454(35,9)$ & 1.379 & $31,3(29,9-32,7)$ \\
\hline Sim & $7.939(64,1)$ & 2.857 & $36,2(35,1-37,3)$ \\
\hline \multicolumn{4}{|c|}{ Morbidade referida } \\
\hline \multicolumn{4}{|c|}{ Hipertensão arterial sistêmica $(n=12.324)$} \\
\hline Não & $10.320(83,7)$ & 3.229 & $31,5(30,6-32,4)$ \\
\hline $\operatorname{Sim}$ & $2.004(16,3)$ & 998 & $50,1(47,9-52,3)$ \\
\hline \multicolumn{4}{|c|}{ Diabetes mellitus ( $n=12.344$ ) } \\
\hline Não & $11.904(96,4)$ & 3.979 & $33,6(32,8-34,5)$ \\
\hline Sim & $440(3,6)$ & 258 & $58,9(54,1-63,5)$ \\
\hline \multicolumn{4}{|c|}{ Problema de nervos $(n=12.349)$} \\
\hline Não & $10.911(88,4)$ & 3.484 & $32,1(31,2-33,0)$ \\
\hline Sim & $1.438(11,6)$ & 753 & $52,8(50,2-55,4)$ \\
\hline \multicolumn{4}{|c|}{ Região geográfica ( $n=12.402$ ) } \\
\hline Centro-oeste & $1.363(11,0)$ & 441 & $32,5(30,0-35,1)$ \\
\hline Norte & $988(8,0)$ & 214 & $21,7(19,1-24,3)$ \\
\hline Nordeste & $2.951(23,8)$ & 916 & $31,2(29,5-32,9)$ \\
\hline Sudeste & $4.391(35,4)$ & 1.593 & $36,8(35,4-38,2)$ \\
\hline Sul & $2.709(21,8)$ & 1.077 & $40,0(38,2-41,9)$ \\
\hline
\end{tabular}

(continua) 
Tabela 1 (continuação)

\begin{tabular}{|c|c|c|c|}
\hline \multirow[t]{2}{*}{ Variável } & \multirow{2}{*}{$\begin{array}{c}\text { Amostra total } \\
n *(\%)\end{array}$} & \multicolumn{2}{|c|}{ Utilização de atendimento médico } \\
\hline & & $\mathrm{n} * \star$ & $\%(I C 95 \%)$ \\
\hline \multicolumn{4}{|c|}{ Porte populacional (habitantes) $(n=12.402)$} \\
\hline$\leq 30.000$ & $2.839(22,9)$ & 978 & $35,2(33,4-37,0)$ \\
\hline $30.001-100.000$ & $1.765(14,2)$ & 561 & $31,8(29,6-34,0)$ \\
\hline $100.001-1.000 .000$ & $4.950(39,9)$ & 1.642 & $33,4(32,1-34,7)$ \\
\hline $1.000 .001 \mathrm{e}+$ & $2.848(23,0)$ & 1.060 & $37,4(35,6-39,2)$ \\
\hline
\end{tabular}

IC95\%: intervalo de 95\% de confiança.

* $N=12.402$;

** $\mathrm{N}=4.241$;

*** $\mathrm{n}$ válido em cada variável.

Aproximadamente dois terços $(64,1 \%)$ viviam com companheiro, $16,3 \%$ referiram ter diagnóstico médico de hipertensão arterial sistêmica, $3,6 \%$ de diabetes e $11,6 \%$ de problemas de nervos. O maior número de entrevistados residia na Região Sudeste e em municípios entre 100.001 e 1.000 .000 de habitantes $(35,4 \%$ e $39,9 \%$, respectivamente) (Tabela 1 ).

A prevalência de atendimento médico ambulatorial nos três meses anteriores à entrevista foi de 34,6\% (IC95\%: 33,7-35,9) ( $\mathrm{n}=4.241$ adultos); as análises a seguir se referem a esse subgrupo.

A utilização de atendimento médico ambulatorial foi maior para o sexo feminino e aumentou linearmente com o incremento da idade. Indivíduos que referiram cor da pele branca, viver com companheiro e possuir alguma morbidade utilizaram mais o atendimento médico ambulatorial. A maior utilização de atendimento médico-ambulatorial ocorreu em indivíduos com diagnóstico de diabetes mellitus (58,9\%) (Tabela 1$)$.

A utilização de atendimento médico-ambulatorial foi significativamente maior entre os adultos das classes econômicas A/B, com 12 anos ou mais de escolaridade, com renda superior a um salário mínimo, residentes na Região Sul (Tabela 1).

Considerando a natureza dos serviços, o SUS foi responsável por mais da metade $(53,6 \%)$ dos atendimentos, algum convênio de saúde foi utilizado por $33,9 \%$ da amostra e os serviços privados por apenas $12,5 \%$. De acordo com o tipo de serviço, as proporções de utilização foram semelhantes entre consultórios e UBS (40,3\% e $39,6 \%$, respectivamente), e apenas $20,1 \%$ utilizaram ambulatórios.

$\mathrm{O}$ atendimento médico pelo SUS foi o que apresentou significativamente maior proporção de utilização, independentemente da região, do porte populacional e da morbidade referida. As maiores diferenças foram observadas na Região
Nordeste e as menores diferenças na Região Sudeste. As classes A e B utilizaram mais atendimento por convênio $(59,4 \%)$ e particular $(15,5 \%)$ e as classes D e E utilizaram mais o SUS $(85,1 \%)$ (Tabela 2).

\section{Características da utilização do atendimento médico ambulatorial de acordo com a natureza do serviço}

Do total de adultos que utilizaram atendimento médico ambulatorial, $13,7 \%$ referiram pagar pelo atendimento. Essa realidade foi mais frequente no atendimento particular $(78,2 \%)$. Os atendimentos ocorreram fora do município para $13,1 \%$ da amostra, sendo maior no atendimento particular (27\%) (Tabela 3).

A prescrição de medicação foi comum a $64,4 \%$ dos entrevistados, com maior ocorrência para indivíduos que consultaram pelo SUS (67,3\%). Dos indivíduos que saíram do atendimento com prescrição, $70 \%$ gastaram dinheiro para comprar algum ou todos os medicamentos, e 75,3\% compraram todos os medicamentos prescritos. A maior proporção de gasto com compra de medicação foi observada entre os adultos que utilizaram o atendimento particular $(96,7 \%)$ (Tabela 3).

Em 42,9\% dos atendimentos houve a solicitação de exames, sendo pouco mais frequente entre os adultos que realizaram o atendimento por convênio $(46,6 \%)$. Um quarto dos entrevistados $(24,9 \%)$ precisou pagar para realizar os exames e, desses, 71,9\% o fizeram na rede particular. Apenas $7,1 \%$ dos entrevistados foram encaminhados para atendimento em outro serviço de saúde, realidade mais frequente para indivíduos que utilizaram o SUS (8,7\%) (Tabela 3$)$.

A maioria dos adultos considerou o tempo de espera e o tempo de atendimento bom ou ótimo $(60,7 \%$ e $88,1 \%$, respectivamente). Ao sair 
Natureza do atendimento médico utilizado de acordo com região geográfica, porte populacional, classe econômica e morbidade referida. Brasil, 2009

\begin{tabular}{|c|c|c|c|c|}
\hline \multirow[t]{2}{*}{ Variável } & \multicolumn{3}{|c|}{ Natureza do serviço * [n (\%)] } & \multirow[t]{2}{*}{ Valor de $p$} \\
\hline & Convênio & Particular & SUS & \\
\hline Região geográfica $(n=4.200)$ ** & & & & $<0,001$ \\
\hline Centro-oeste & $152(34,6)$ & $49(11,1)$ & $239(54,3)$ & \\
\hline Norte & $55(25,7)$ & $39(18,2)$ & $120(56,1)$ & \\
\hline Nordeste & $168(18,4)$ & $127(13,9)$ & $616(67,7)$ & \\
\hline Sudeste & $658(41,9)$ & $174(11,1)$ & $739(47,0)$ & \\
\hline Sul & $393(36,9)$ & $134(12,6)$ & $537(50,5)$ & \\
\hline Porte populacional (habitantes) $(n=4.200)$ & & & & $<0,001$ \\
\hline$\leq 30.000$ & $180(18,8)$ & $107(11,2)$ & $671(70,0)$ & \\
\hline $30.001-100.000$ & $125(22,6)$ & $87(15,7)$ & $341(61,7)$ & \\
\hline 100.001-1.000.000 & $667(40,8)$ & $219(13,4)$ & $750(45,8)$ & \\
\hline $1.000 .001 \mathrm{e}+$ & $454(43,1)$ & $110(10,5)$ & $489(46,4)$ & \\
\hline Classe econômica $(n=3.963)$ & & & & $<0,001$ \\
\hline $\mathrm{A} / \mathrm{B}$ & $788(59,4)$ & $206(15,6)$ & $332(25,0)$ & \\
\hline C & $518(26,3)$ & $234(11,9)$ & $1.216(61,8)$ & \\
\hline $\mathrm{D} / \mathrm{E}$ & $47(7,0)$ & $53(7,9)$ & $569(85,1)$ & \\
\hline Hipertensão arterial sistêmica $(n=4.186)$ & & & & $<0,001$ \\
\hline Não & $1.147(35,8)$ & $422(13,2)$ & $1.630(51,0)$ & \\
\hline $\operatorname{Sim}$ & $274(27,8)$ & $99(10,0)$ & $614(62,2)$ & \\
\hline Diabetes mellitus ( $n=4.196$ ) & & & & 0,001 \\
\hline Não & $1.356(34,4)$ & $500(12,7)$ & $2.084(52,9)$ & \\
\hline $\operatorname{Sim}$ & $67(26,2)$ & $23(9,0)$ & $166(64,8)$ & \\
\hline Problema de nervos $(n=4.196)$ & & & & $<0,001$ \\
\hline Não & $1.236(35,8)$ & $431(12,5)$ & $1.788(51,7)$ & \\
\hline $\operatorname{Sim}$ & $189(25,5)$ & $92(12,4)$ & $460(62,1)$ & \\
\hline Total & $1.426(33,9)$ & $523(12,5)$ & $2.251(53,6)$ & \\
\hline
\end{tabular}

SUS: Sistema Único de Saúde.

* Análise restrita a 4.200 adultos que utilizaram atendimento médico;

** $\mathrm{n}$ válido em cada variável.

do atendimento com o médico, $26 \%$ dos adultos referiram não ter recebido explicação sobre o motivo que o fez buscar o atendimento e, $32,2 \%$ dos entrevistados não observaram nenhuma melhora do problema (Tabela 3 ).

A maior proporção de motivos referidos para a utilização de atendimento médico nos três meses anteriores à entrevista foi para "revisar/ acompanhar problema" (45,5\%), independentemente da natureza do serviço. Tendo em conta a natureza do serviço, o convênio foi mais utilizado para a realização de check-up $(35,2 \%)$ e exame de próstata (48\%). O SUS se destacou em renovar receita de medicamentos, realizar pré-natal e revisar e acompanhar problema (Figura 1).

\section{Discussão}

A utilização de atendimento médico conforme a natureza dos serviços é uma das ênfases do presente estudo. O SUS foi responsável pela maioria dos atendimentos realizados, apesar da participação importante dos planos de saúde, especialmente para alguns procedimentos.

A prevalência de utilização de atendimento médico-ambulatorial nos três meses anteriores à entrevista foi de $34,6 \%$, variando de acordo com a natureza do atendimento, de $12,5 \%$ (particular) a 53,6\% (SUS). Esse achado, relativo à utilização global, foi compatível com os encontrados na Pesquisa Nacional por Amostras de Domicílios (PNAD) de 1998 (57,8\%), 2003 (64,7\%) e 2008 $(69,6 \%)$ para a mesma faixa etária e residentes 
Características da utilização do atendimento médico-ambulatorial. Brasil, 2009

\begin{tabular}{|c|c|c|c|c|c|}
\hline \multirow[t]{2}{*}{ Variável } & \multirow[t]{2}{*}{ n (\%) } & \multicolumn{3}{|c|}{ Natureza do serviço * [n (\%)] } & \multirow[t]{2}{*}{ Valor de $\mathrm{p}$} \\
\hline & & Convênio & Particular & SUS & \\
\hline Gastou com o último atendimento $(n=4.195) \star \star$ & $573(13,7)$ & $159(11,2)$ & $408(78,2)$ & $6(0,3)$ & $<0,001$ \\
\hline Foi atendido fora do município de residência $(n=4.194)$ & $549(13,1)$ & $274(19,3)$ & $141(27,0)$ & $134(6,0)$ & $<0,001$ \\
\hline Foi prescrita medicação $(n=4.150)$ & $2.671(64,4)$ & $834(59,6)$ & $334(64,7)$ & $1.503(67,3)$ & $<0,001$ \\
\hline Teve gastos com medicação $(n=2.655)$ & $1.856 * \star \star(69,9)$ & $783(94,6)$ & $321(96,7)$ & $752(50,3)$ & $<0,001$ \\
\hline Saiu do atendimento com pedido de exame $(n=4.156)$ & $1.783(42,9)$ & $654(46,6)$ & $227(44,0)$ & $902(40,4)$ & 0,001 \\
\hline Teve gastos com exames $(n=1.341)$ & $334(24,9)$ & $96(18,3)$ & $133(71,9)$ & $105(16,7)$ & $<0,001$ \\
\hline Foi encaminhado para outro serviço $(n=4.175)$ & $297(7,1)$ & $81(5,7)$ & $21(4,0)$ & $195(8,7)$ & $<0,001$ \\
\hline Satisfação com o tempo de espera $(n=4.008)$ & & & & & $<0,001$ \\
\hline Péssimo & $548(13,7)$ & $109(8,2)$ & $23(4,7)$ & $416(19,1)$ & \\
\hline Ruim & $309(7,7)$ & $82(6,1)$ & $32(6,5)$ & $195(9,0)$ & \\
\hline Regular & $718(17,9)$ & $233(17,4)$ & $73(14,8)$ & $412(18,9)$ & \\
\hline Bom & $1.861(46,4)$ & $662(49,5)$ & $251(51,0)$ & $948(43,5)$ & \\
\hline Ótimo & $572(14,3)$ & $251(18,8)$ & $113(23,0)$ & $208(9,5)$ & \\
\hline Satisfação com o atendimento $(n=4.025)$ & & & & & $<0,001$ \\
\hline Péssimo & $107(2,7)$ & $18(1,3)$ & $5(1,0)$ & $84(3,9)$ & \\
\hline Ruim & $61(1,5)$ & $7(0,5)$ & $6(1,2)$ & $48(2,2)$ & \\
\hline Regular & $313(7,8)$ & $60(4,5)$ & $29(5,8)$ & $224(10,3)$ & \\
\hline Bom & $2.169(53,9)$ & $707(52,3)$ & $212(42,7)$ & $1.250(57,4)$ & \\
\hline Ótimo & $1.375(34,2)$ & $559(41,4)$ & $245(49,3)$ & $571(26,2)$ & \\
\hline $\begin{array}{l}\text { Recebeu explicação sobre o motivo pelo qual buscou o } \\
\text { atendimento }(n=4.100)\end{array}$ & $3.033(74,0)$ & $1.105(80,3)$ & $402(79,5)$ & $1.526(68,9)$ & $<0,001$ \\
\hline O que aconteceu após o atendimento $(n=3.997)$ & & & & & $<0,001$ \\
\hline Piorou & $41(1,0)$ & $6(0,5)$ & $6(1,2)$ & $29(1,3)$ & \\
\hline Continua como antes & $1.292(32,2)$ & $452(33,8)$ & $131(26,6)$ & $709(32,7)$ & \\
\hline Melhorou um pouco & $848(21,2)$ & $261(19,5)$ & $93(18,9)$ & $494(22,8)$ & \\
\hline Melhorou bastante & $1.045(26,1)$ & $333(24,9)$ & $145(29,5)$ & $567(26,2)$ & \\
\hline Curou/Resolveu & $771(19,3)$ & $286(21,3)$ & $117(23,8)$ & $368(17,0)$ & \\
\hline
\end{tabular}

SUS: Sistema Único de Saúde.

* Análise restrita a 4.200 adultos que utilizaram atendimento médico;

** $\mathrm{n}$ válido em cada variável;

*** Análise refere-se a 1.856 adultos que não conseguiram a medicação pelo SUS.

em áreas urbanas. Em estudo comparativo realizado no município de Pelotas (Rio Grande do Sul) as prevalências encontradas para o ano de 1992 e 2007 foram de $69,8 \%$ e $76,2 \%$ para o período dos últimos 12 meses, respectivamente, e para os últimos três meses, de 39,5\% e 60,6\%.

Além do intervalo de tempo transcorrido entre o evento e a coleta de dados, a importância atribuída pelo entrevistado ao episódio ou evento também pode afetar a informação sobre o uso de serviços de saúde 24,25. Para este estudo, não foram coletados dados de percepção dos indivíduos sobre a relevância daquele episódio ou daquele problema de saúde que motivaram o atendimento médico.
Independentemente de distintos períodos recordatórios, os resultados deste estudo foram similares aos de outros inquéritos no que se refere aos diferenciais da utilização de serviços. As mulheres, os indivíduos com mais idade, aqueles que referiram cor da pele branca, com mais anos de escolaridade, com maior renda, de classe econômica A ou B, que vivem com companheiro e com doenças crônicas referiram utilizar mais o atendimento médico.

Considerando as variáveis demográficas, evidências apontam para uma maior utilização do atendimento médico por mulheres, particularmente em razão das características do gênero (início da idade reprodutiva, controle da fecun- 


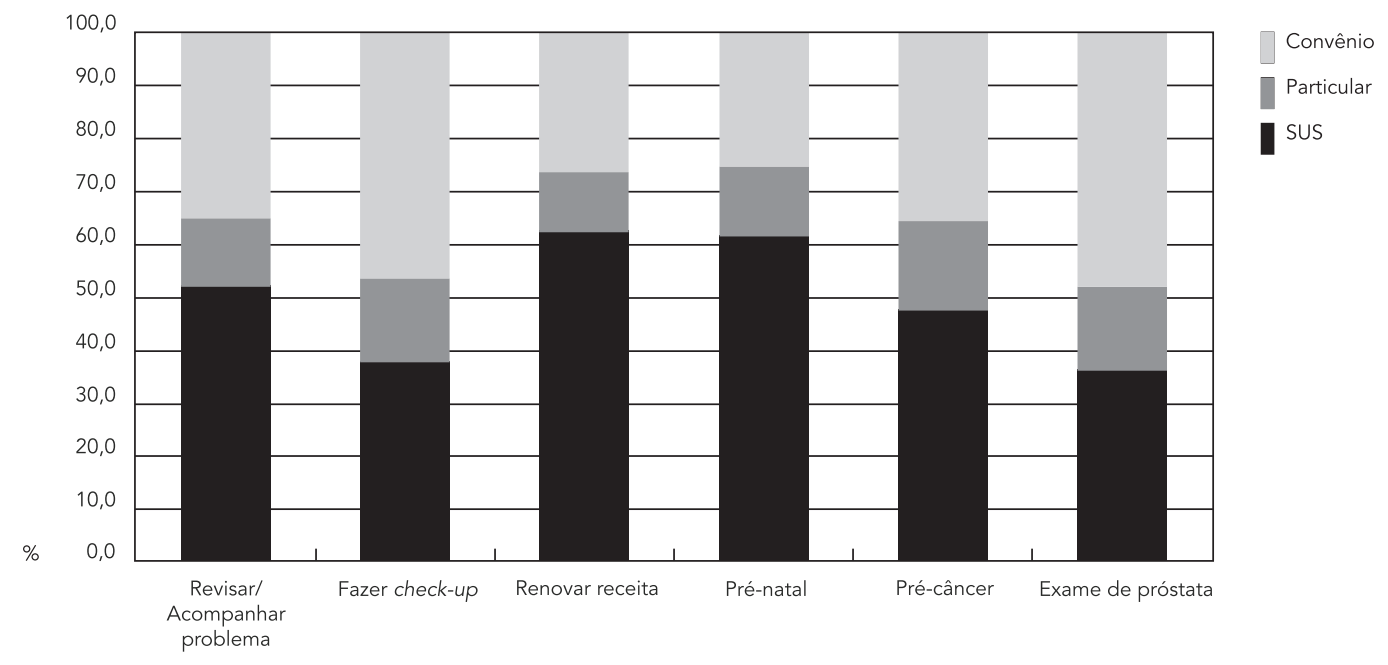

SUS: Sistema Único de Saúde

didade e exames ginecológicos preventivos - citopatológico, mamografia) 24,26,27,28,29,30,31,32,33, diferentes fatores de exposição ao risco de adoecimento (tipo de trabalho, dos hábitos de vida $\mathrm{e}$ de consumo) 28,30,31,32,33,34, maior interesse pela sua condição de saúde 28,31,32,32,33,34 e "feminização" do envelhecimento 28,35. Estudo qualitativo realizado no Rio de Janeiro em 2004 destaca que os homens procuram menos os serviços de saúde porque entendem o cuidar como uma tarefa feminina e relatam a dificuldade de acesso aos serviços (horário de funcionamento, tempo de espera e precarização) 36 , além de expressarem e perceberem a saúde e doença de forma diferenciada 4 .

Indivíduos com mais idade utilizam mais os serviços de saúde, pois as necessidades em saúde variam de acordo com as fases do ciclo de vida, sendo mais evidentes nos primeiros anos de vida e nas idades mais avançadas. Observa-se maior frequência de doenças agudas na infância e de doenças crônicas nos idosos, associadas às transformações biológicas e fisiológicas que promovem a variação na necessidade de cuidado médico por apresentarem múltiplas morbidades $4,13,26,27,28,30,35$.

O tamanho da família e a cor da pele podem influenciar a maior ou menor utilização dos serviços de saúde. Frequentemente a cor da pele e a estrutura familiar estão associadas à classe econômica, mostrando correlação positiva entre o acesso ao médico e o poder aquisitivo do indivíduo, determinando o sentido do efeito da utilização do serviço de saúde $24,27,31$. Neste estudo se observou que indivíduos que vivem com companheiro e se autodeclararam brancos utilizaram mais os serviços de saúde.

Aproximadamente dois terços da carga mundial de doenças se devem às condições crônicas não transmissíveis, podendo atingir $80 \%$ em países em desenvolvimento até o ano de 2020. Além das doenças não transmissíveis, como o diabetes, o câncer e as doenças cardiovasculares, incluem-se como crônicas as doenças transmissíveis persistentes, como a AIDS e as hepatites; os distúrbios mentais de longo prazo, como a depressão e a demência; e as deficiências físicas, como as amputações, a cegueira e os transtornos das articulações 37. Logo, é compreensível que indivíduos que declaram diagnóstico médico de uma condição crônica de saúde utilizem mais os serviços de saúde.

Em estudo realizado por Capilheira \& Santos 38 , observou-se que a presença de hipertensão arterial sistêmica aumentava a probabilidade de consultar um médico e de "superutilizar" os serviços de saúde.

O envelhecimento e a presença de doenças crônicas mostram-se fortemente associados, pois à medida que os indivíduos envelhecem, as doenças crônicas ainda se transformam nas primeiras causas de morbidade, incapacidade e mortalidade, aumentando a necessidade de utilizar os serviços de saúde 35,39. 
Em um cenário mais otimista, poder-se-ia pensar que, a exemplo do que é sugerido pela teoria da compressão da morbidade, o incremento das políticas públicas com ênfase em programas de saúde preventivos, o aumento de recursos diagnósticos e terapêuticos eficazes, a mudança de comportamento (incentivo à atividade física) e a melhoria na qualidade de vida (hábitos de vida saudáveis) acarretariam um aumento da expectativa de vida com declínio das morbidades 40 . Assim, os idosos viveriam mais tempo com menor carga de doenças, tanto no que diz respeito à ocorrência quanto à gravidade. Nessa hipótese não haveria necessariamente uma diminuição da utilização de serviços médicos, mas eventualmente uma mudança do perfil de demanda, que poderia estar mais dirigido a ações de promoção da saúde e de prevenção de agravos.

A literatura tem apontado que pessoas de maior poder aquisitivo e maior escolaridade têm utilizado mais os serviços de saúde de modo geral e também recebido mais atendimento médico, gerando possíveis situações de iniquidade 41 . A escolaridade se associa a um maior conhecimento e melhor comportamento quanto à saúde e pode ser considerada um importante preditor de utilização de serviços de saúde por conta de sua relação com melhores condições sociais e financeiras 26,28,29.

Da mesma forma, sabe-se que pessoas com melhor condição socioeconômica têm maior probabilidade de receber cuidado de um especialista e utilizar mais os serviços ambulatoriais, independentemente das condições prévias de saúde, em comparação aos mais pobres 26,27,42. Indivíduos com menor escolaridade, renda e informação estão sujeitos a situações de iniquidade, utilizando com menor frequência os serviços de saúde ("Lei dos cuidados inversos") 43.

Apesar de não haver uma diferença estatisticamente significativa de acordo com a região geográfica, verifica-se uma tendência de maior utilização do atendimento médico de acordo com o grau de desenvolvimento socioeconômico da região, pois maiores proporções do desfecho foram registradas nas regiões Sul e Sudeste 44 .

A utilização de atendimento médico-ambulatorial foi maior nos municípios pequenos e nos de maior porte, apesar de não ter se encontrado diferença estatisticamente significativa. Tal achado pode estar relacionado à maior disponibilidade de consultas, já que os padrões de utilização de serviços estão intimamente relacionados com a disponibilidade, facilidade de acesso e modelos de atenção à saúde 24 .

Como esperado, a utilização do atendimento médico-ambulatorial variou de acordo com a natureza do serviço, tendo sido mais prevalente em serviços do SUS, independentemente da região geográfica, porte populacional e classe econômica dos entrevistados. De acordo com dados da PNAD de 1998, considerando o atendimento nas duas semanas anteriores à entrevista, 35,8\% dos indivíduos utilizaram plano de saúde, $15,8 \%$ pagaram algum valor pelo atendimento e $49,3 \%$ utilizaram o SUS. Na PNAD de $2003,26 \%$ dos indivíduos utilizaram plano de saúde, $14,8 \%$ pagaram pelo atendimento e $56,5 \%$ utilizaram o SUS. Na PNAD de 2008, 26,2\% dos indivíduos utilizaram plano de saúde, $18,7 \%$ pagaram pelo atendimento e $57,2 \%$ utilizaram o SUS. Portanto, observa-se que entre o período de 1998 a 2008 houve um incremento na utilização de serviços do SUS e de serviços pagos diretamente e um decréscimo na utilização de planos de saúde. Tais mudanças poderiam ser parcialmente atribuídas ao aumento da cobertura de serviços públicos na atenção básica, especialmente com a expansão da Estratégia Saúde da Família 45,46,47,48,49.

Diferentemente da PNAD, neste estudo o pagamento pelo atendimento foi investigado de forma independente da natureza do serviço utilizado, o que resultou em, por exemplo, 21,8\% dos que utilizaram serviços privados não terem pago por eles. É provável que parte dos atendimentos particulares possam ter sido retornos de consultas prévias, o que não implicaria pagamentos, uma vez que a pergunta se referia ao último contato com os serviços. Outra possibilidade seriam os atendimentos prestados por familiares e amigos.

Entre os motivos referidos para a utilização do atendimento médico, destacou-se "revisar/ acompanhar problema" (45,5\%), resultado similar ao da PNAD de 2003 e 2008, em que a maior procura por atendimento nos serviços de saúde foi motivada pela presença de doença $(51,9 \%$ e $50 \%$, respectivamente), enquanto na PNAD de 1998 os atendimentos de rotina ou prevenção $(37,3 \%)$ foram os mais frequentes.

Os resultados descritivos da utilização de atendimento médico ambulatorial de acordo com a natureza do serviço destacam que usuários do SUS gastaram menos com o último atendimento, com as medicações e os exames; tiveram que sair menos do município; tiveram que ser mais encaminhados para outros serviços; receberam menos informações sobre seu problema; saíram do atendimento com mais prescrição de medicamentos e menos de exames complementares; referiram menos satisfação com tempo de espera e com o atendimento de forma geral.

Sem considerar a natureza do serviço, o atendimento foi avaliado como "bom" ou “ótimo" pela maioria dos entrevistados $(88,1 \%)$. Na PNAD, 
os entrevistados classificaram o atendimento recebido como "bom" ou "muito bom" em níveis bastante similares: $86,2 \%$ (PNAD 1998), $86 \%$ (PNAD 2003) e $86,4 \%$ (PNAD 2008).

O uso do delineamento transversal impossibilita estabelecer relações temporais de causa e efeito 50 . No caso deste estudo, não foi possível determinar se quem mais frequenta os serviços de saúde são os indivíduos mais doentes por serem mais diagnosticados, ou, se quem é realmente mais doente utiliza mais os serviços por causa da presença prévia de doenças.

A utilização de diferentes períodos recordatórios dificulta a comparação dos achados. A PNAD utiliza 12 meses em seus inquéritos; esse período maior pode ter sido responsável pelas maiores prevalências. Períodos mais extensos podem fornecer estimativas pouco precisas, levando a uma subenumeração de episódios. Por outro lado, períodos muito curtos poderiam não ser suficientes para incluir um número razoável de eventos e/ou necessidades relacionadas ao agravo, implicando uma subestimativa mais pronunciada. Além disso, essa decisão exigiria maiores tamanhos de amostra, podendo inviabilizar a realização de estudos 38,51 .

Entre os pontos positivos, destaca-se a coleta de dados primários, a baixa taxa de não resposta, o tamanho e abrangência da amostra com representatividade nacional. Essa representatividade foi fortalecida ao comparar com os dados do censo de 2010, no qual se encontrou uma similaridade na distribuição por idade e sexo entre os dois inquéritos de abrangência nacional.

\section{Conclusão}

Os padrões de utilização de serviços de saúde continuam socialmente determinados, resultando da oferta, das características sociodemográficas e do perfil de saúde dos usuários 13,26,27,52. A comparação dos achados com outros inquéritos de abrangência nacional permite verificar mudanças importantes nos últimos anos no Brasil: os serviços públicos e os serviços pagos diretamente pelos usuários vêm aumentando, com a redução dos serviços pagos por intermédio de convênios. O artigo também destacou a distribuição de indicadores selecionados de qualidade da atenção ambulatorial de acordo com a natureza do serviço, como por exemplo, observou-se que o SUS responde por menor satisfação dos usuários com o tempo de espera, mas pela maior satisfação com os atendimentos.

Essas informações podem subsidiar o planejamento dos serviços, organizar a oferta e identificar barreiras de acesso, considerando que o atendimento médico permite realizar intervenções preventivas relacionadas com mudança de hábitos de vida, realização de atividades saudáveis e rastreamento de doenças em períodos assintomáticos 53 . 


\section{Resumen}

Indicadores de la oferta, acceso y uso son útiles para caracterizar los servicios, las actividades de planificación y organización de la demanda. El estudio describe los patrones de uso de tratamiento ambulatorio y su asociación con variables sociodemográficas, morbilidad, tamaño del municipio y de la región, de acuerdo con la naturaleza jurídica del servicio. Se trata de una base poblacional transversal 12.402 adultos brasileños, entre 20 y 59 años, que viven en zonas urbanas de 100 municipios en cinco regiones. La prevalencia de tratamiento ambulatorio en los tres meses anteriores a la entrevista fue del 34,6\%. El Sistema Único de Salud fue responsable de más de la mitad (53,6\%) de los casos, un plan de salud fue utilizado por el 34\% de la muestra y los servicios privados representaron el 12,4\%, con independencia de la región, el tamaño de la población y la morbilidad anteriormente. Los patrones de uso de los servicios de salud siguen siendo socialmente determinados, lo que resulta en la oferta, el perfil sociodemográfico y la salud de los usuarios.

Atención Ambulatoria; Sistema Único de Salud; Evaluación en Salud

\section{Colaboradores}

A. S. Dilélio, E. Tomasi e L. A. Facchini participaram de todas as etapas do artigo, desde a concepção até a redação final. E. Thumé, D. S. Silveira, F. C. V. Siqueira, R. X. Piccini, S. M. Silva e B. P. Nunes contribuíram nas etapas de revisão bibliográfica e revisão do artigo.

\section{Agradecimentos}

O estudo foi financiado pelo Ministério da Saúde.

\section{Referências}

1. Departamento de Regulação, Avaliação e Controle de Sistemas, Secretaria de Atenção à Saúde, Ministério da Saúde. Programa Nacional de Avaliação de Serviços de Saúde. Brasília: Ministério da Saúde; 2004/2005.

2. Travassos C, Castro M. Determinantes e desigualdades sociais no acesso e na utilização de serviços de saúde. In: Giovanella L, Escorel S, Lobato LVC, Noronha JC, Carvalho AI, organizadores. Políticas e sistema de saúde no Brasil. Rio de Janeiro: Editora Fiocruz; 2008. p. 215-46.

3. Souza LEPF, Contandriopoulos A-P. O uso de pesquisas na formulação de políticas de saúde: obstáculos e estratégias. Cad Saúde Pública 2004; 20:546-54.

4. Travassos C, Viacava F, Pinheiro R, Brito A. Utilização dos serviços de saúde no Brasil: gênero, características familiares e condição social. Rev Panam Salud Pública 2002; 11:365-73.

5. Travassos C, Viacava F. Utilização e financiamento de serviços de saúde: dez anos de informação das PNAD. Ciênc Saúde Coletiva 2011; 16:3646-7.

6. Valderas JM, Starfield B, Sibbald B, Salisbury C, Roland M. Defining comorbidity: implications for understanding health and health services. Ann Fam Med 2009; 7:357-63.

7. Facchini LA, Piccini RX, Tomasi E, Thumé E, Teixeira VA, Silveira DS, et al. Avaliação de efetividade da Atenção Básica à Saúde em municípios das regiões Sul e Nordeste do Brasil: contribuições metodológicas. Cad Saúde Pública 2008; 24 Suppl 1:S159-72.

8. Tomasi E, Facchini LA, Thumé E, Piccini RX, Osorio A, Silveira DS, et al. Characteristics of primary healthcare service use in the southern and northeastern regions of Brazil: differences by care model. Ciênc Saúde Coletiva 2011; 16:4395-404.

9. Junqueira TS, Cotta RMM, Gomes RC, Silveira SFR, Siqueira-Batista R, Pinheiro TMM, et al. As relações laborais no âmbito da municipalização da gestão em saúde e os dilemas da relação expansão/precarização do trabalho no contexto do SUS. Cad Saúde Pública 2010; 26:918-28.

10. Giovanella L, Mendonça MHM, Almeida PF, Escorel S, Senna MCM, Fausto MCR, et al. Saúde da família: limites e possibilidades para uma abordagem integral de atenção primária à saúde no Brasil. Ciênc Saúde Coletiva 2009; 14:783-94. 
11. Piccini RX, Facchini LA, Tomasi E, Thumé E, Silveira DS, Siqueira FV, et al. Necessidades de saúde comuns aos idosos: efetividade na oferta e utilização em atenção básica à saúde. Ciênc Saúde Coletiva 2006; 11:657-67.

12. Siqueira FCV, Facchini LA, Silveira DS, Piccini RX, Thumé E, Tomasi E. Barreiras arquitetônicas a idosos e portadores de deficiência física: um estudo epidemiológico da estrutura física das unidades básicas de saúde em sete estados do Brasil. Ciênc Saúde Coletiva 2009; 14:39-44.

13. Ribeiro MCSA, Barata RB, Almeida MF, Silva ZP. Perfil sociodemográfico e padrão de utilização de serviços de saúde para usuários e não usuários do SUS-PNAD 2003. Ciênc Saúde Coletiva 2006; 11:1011-22.

14. Ministério da Saúde. Portaria GM/MS no 1.654, de 19 de julho de 2011. Institui, no âmbito do Sistema Único de Saúde, o Programa Nacional de Melhoria do Acesso e da Qualidade da Atenção Básica (PMAQ-AB) e o Incentivo Financeiro do PMAQ-AB, denominado Componente de Qualidade do Piso de Atenção Básica Variável - PAB Variável. Diário Oficial da União 2011; 20 jul.

15. Bonfada D, de Paiva Cavalcante JRL, de Araujo DP, Guimarães J. A integralidade da atenção à saúde como eixo da organização tecnológica nos serviços. Ciênc Saúde Coletiva 2012; 17:555-60.

16. Conselho Nacional de Secretarias Municipais de Saúde. Reflexões aos novos gestores municipais de saúde. Brasília: Conselho Nacional de Secretarias Municipais de Saúde; 2009.

17. Secretaria de Vigilância em Saúde/Secretaria de Atenção à Saúde, Ministério da Saúde. Política Nacional de Promoção da Saúde. Brasília: Ministério da Saúde; 2006.

18. Ministério da Saúde. Portaria GM/MS no 648, de 28 de março de 2006. Aprova a Política Nacional da Atenção Básica, estabelecendo a revisão de diretrizes e normas para a organização da Atenção Básica para o Programa Saúde da Família (PSF) e o Programa Agentes Comunitários de Saúde (PACS). Diário Oficial da União 2006; 29 mar.

19. Szwarcwald CL, Damacena GN. Amostras complexas em inquéritos populacionais: planejamento e implicações na análise estatística dos dados. Rev Bras Epidemiol 2008; 11 Suppl 1:38-45.

20. Instituto Brasileiro de Geografia e Estatística. Pesquisa Nacional por Amostra de Domicílios: síntese de indicadores, 2003. Rio de Janeiro: Instituto Brasileiro de Geografia e Estatística; 2003.

21. Piccini RX, Facchini LA, Tomasi E, Siqueira FV, Silveira DS, Thumé E, et al. Promotion, prevention and arterial hypertension care in Brazil. Rev Saúde Pública 2012; 46:543-50.

22. Silva SM, Facchini LA, Tomasi E, Piccini R, Thumé E, Silveira DS, et al. Recebimento de orientação sobre consumo de sal, açúcar e gorduras em adultos: um estudo de base nacional. Rev Bras Epidemiol 2013; 16:995-1004.

23. Madeira MC, Siqueira FCV, Facchini LA, Silveira DS, Tomasi E, Thumé E, et al. Atividade física no deslocamento em adultos e idosos do Brasil: prevalências e fatores associados. Cad Saúde Pública 2013; 29:165-74.
24. Bastos GAN, Santos IS, Costa JSD, Capilheira MF. Ambulatory care utilization in the past 15 years: comparison between two population-based studies. Rev Bras Epidemiol 2011; 14:620-32.

25. Ross DA, Vaughan JP. Health interview surveys in developing countries: a methodological review. Stud Fam Plann 1986; 17:78-94.

26. Dias da Costa JS, Facchini LA. Utilização de serviços ambulatoriais em Pelotas: onde a população consulta e com que frequência. Rev Saúde Pública 1997; 31:360-9.

27. Mendoza-Sassi R, Béria JU. Utilización de los servicios de salud: una revisión sistemática sobre los factores relacionados. Cad Saúde Pública 2001; 17:819-32.

28. Pinheiro RS, Viacava F, Travassos C, Brito AS. Gênero, morbidade, acesso e utilização de serviços de saúde no Brasil. Ciênc Saúde Coletiva 2002; 7:687-707.

29. Mendoza-Sassi R, Beria JU, Barros AJD. Outpatient health service utilization and associated factors: a population-based study. Rev Saúde Pública 2003; 37:372-8.

30. Turci MA, Lima-Costa MF, Proietti FA, Cesar CC, Macinko J. Intraurban differences in the use of ambulatory health services in a large Brazilian city. J Urban Health 2010; 87:994-1006.

31. Instituto Brasileiro de Geografia e Estatística. Pesquisa Nacional por Amostra de Domicílios: acesso e utilização dos serviços de saúde, 1998. Rio de Janeiro: Instituto Brasileiro de Geografia e Estatística; 1998.

32. Instituto Brasileiro de Geografia e Estatística. Pesquisa Nacional por Amostra de Domicílios: utilização dos serviços de saúde, 2003. Rio de Janeiro: Instituto Brasileiro de Geografia e Estatística; 2003.

33. Instituto Brasileiro de Geografia e Estatística. Pesquisa Nacional por Amostra de Domicílios. Um panorama da saúde no Brasil: acesso e utilização dos serviços, condições de saúde e fatores de risco e proteção à saúde, 2008. Rio de Janeiro: Instituto Brasileiro de Geografia e Estatística; 2008.

34. Verbrugge LM. The twain meet: empirical explanations of sex differences in health and mortality. J Health Soc Behav 1989; 30:282-304.

35. Lima-Costa MF. Epidemiologia do envelhecimento no Brasil. In: Rouquayrol MZ, Almeida Filho N, organizadores. Epidemiologia e saúde. Rio de Janeiro: MEDSI; 2003. p. 499-514.

36. Gomes R, Nascimento EF, Araújo FC. Por que os homens buscam menos os serviços de saúde do que as mulheres? As explicações de homens com baixa escolaridade e homens com ensino superior. Cad Saúde Pública 2007; 23:565-74.

37. Organização Mundial da Saúde. Cuidados inovadores para condições crônicas: componentes estruturais de ação: relatório mundial. Brasília: Organização Mundial da Saúde; 2003.

38. Capilheira MF, Santos IS. Fatores individuais associados à utilização de consultas médicas por adultos. Rev Saúde Pública 2006; 40:436-43.

39. Thumé E, Facchini LA, Wyshak G, Campbell P. The utilization of home care by the elderly in Brazil's primary health care system. Am J Public Health 2011; 101:868-74. 
40. Parker MG, Thorslund M. Health trends in the elderly population: getting better and getting worse. Gerontologist 2007; 47:150-8.

41. Neri M, Soares W. Desigualdade social e saúde no Brasil. Cad Saúde Pública 2002; 18:77-87.

42. Curtis L, MacMinn WJ. Health care utilization in Canada: 25 years of evidence. Hamilton: SEDAP Research Program, McMaster University; 2007. (SEDAP Research Paper, 190).

43. Hart JT. The inverse care law. Lancet 1971; 1:405-12.

44. Travassos C, Oliveira EXG, Viacava F. Desigualdades geográficas e sociais no acesso aos serviços de saúde no Brasil: 1998 e 2003. Ciênc Saúde Coletiva 2006; 11:975-86.

45. Pereira CRS, Roncalli AG, Cangussu MCT, Noro LRA, Patricio AAR, Lima KC. Impacto da Estratégia Saúde da Família sobre indicadores de saúde bucal: análise em municípios do Nordeste brasileiro com mais de 100 mil habitantes. Cad Saúde Pública $2012 ; 28: 449-62$.

46. Oliveira LS, Almeida LGN, Oliveira MAS, Gil GB, Alcione Brasileiro Oliveira C, Medina MG, et al. Acessibilidade a atenção básica em um distrito sanitário de Salvador. Ciênc Saúde Coletiva 2012; 17:3047-56.

47. Macinko J, Lima-Costa MF. Access to, use of and satisfaction with health services among adults enrolled in Brazil's Family Health Strategy: evidence from the 2008 National Household Survey. Trop Med Int Health 2012; 17:36-42.
48. Bousquat A, Gomes A, Alves MCGP. Acesso realizado ao Programa de Saúde da Família em área com "alta" cobertura do subsistema privado. Ciênc Saúde Coletiva 2012; 17:2913-21.

49. Reis RS, Coimbra LC, Silva AA, Santos AM, Alves MT, Lamy ZC, et al. Access to and use of the services of the family health strategy from the perspective of managers, professionals and users. Ciênc Saúde Coletiva 2013; 18:3321-31.

50. Klein $\mathrm{CH}$, Bloch KV. Estudos seccionais. In: Medronho RA, Carvalho DM, Bloch KV, Luiz RR, Werneck GL, organizadores. Epidemiologia. São Paulo: Atheneu; 2004. p. 151-60.

51. Richards SH, Coast J, Peters TJ. Patient reported use of health service resources compared with information from health providers. Health Soc Care Community 2003; 11:510-8.

52. Facchini LA, Piccini RX, Tomasi E, Thumé E, Silveira DS. Projeto de monitoramento e avaliação do Programa de Expansão e Consolidação do Saúde da Família (PROESF): relatório. Pelotas: Universidade Federal de Pelotas; 2006.

53. Stein A, Zelmanowicz AM, Lima AK. Promoção da saúde e detecção precoce de doenças no adulto. In: Duncan BB, Schmidt MI, Giugliani ERJ, organizadores. Medicina ambulatorial: condutas de atenção primária baseadas em evidências. Porto Alegre: Artmed; 2006. p. 567-75.

Recebido em 19/Jun/2013

Versão final reapresentada em 13/Mai/2014

Aprovado em 24/Jun/2014 